\title{
Bloch-Kato conjecture and motivic cohomology with finite coefficients ${ }^{1}$
}

\author{
A. Suslin V. Voevodsky.
}

\section{Contents}

$1 \quad$ Introduction. . . . . . . . . . . . . . . 1

2 Motivic cohomology. ............... 2

3 Motivic cohomology and Milnor's K-theory. . . . . . . . . 10

4 Bloch-Kato conjecture. . . . . . . . . . . . . . . . 14

5 The truncated etale cohomology and Beilinson-Lichtenbaum conjecture. . . . . . . . . . . . . . . . . 17

6 Main theorem. . . . . . . . . . . . . . . . 22

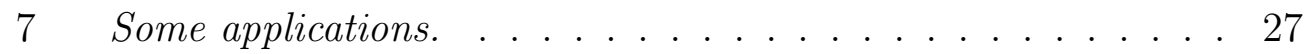

\section{Introduction.}

In this paper we show that the Beilinson-Lichtenbaum conjecture which describes motivic cohomology of (smooth) varieties with finite coefficients in terms of etale cohomology is equivalent to the Bloch-Kato conjecture. Since the later conjecture is known in weight $\leq 2$ (and in weight 3 for $\mathbf{Z} / 2$ coefficients) we deduce from this result some corollaries including the complete description of motivic cohomology with finite coefficients for varieties of dimension $\leq 3$ over algebraically closed fields.

The paper is organized as follows. In the first two sections we remind the construction of motivic cohomology based on the techniques developed in [16] and [5] and establish their relation to Milnor K-theory. In the next two sections the Bloch-Kato and Beilinson-Lichtenbaum conjectures are described and in Section 6 our main theorem (Theorem 5.9) is proven. In the final section we apply this theorem to the cases when the Bloch-Kato conjecture is known obtaining some new results on motivic cohomology with finite coefficients.

\footnotetext{
${ }^{1}$ Preliminary version, June 1995
} 
Since we use the theory of motivic cohomology developed in [5],[16] most of our results (including Theorem 5.9) only hold for fields with resolution of singularities, i.e. at the present moment only in characteristic zero.

\section{Motivic cohomology.}

In this section we recall the construction of motivic cohomology given in [5], [16]. The only new result presented here is a direct proof of the fact that motivic cohomology $H^{n}(k, \mathbf{Z}(n))$ of a field $k$ are isomorphic to Milnor's K-groups $K_{n}^{M}(k)$.

Let $k$ be a field and $S m / k$ the category of smooth schemes over $k$. Recall that a presheaf $F$ on $S m / k$ is called homotopy invariant if for any smooth scheme $U$ the homomorphism $F(U) \rightarrow F\left(U \times \mathbf{A}^{1}\right)$ is an isomorphism.

Consider the standard cosimplicial object $\Delta^{\bullet}$ in $S m / k$. For a presheaf of abelian groups $F$ on $S m / k$ denote by $\underline{C}_{*}(F)$ the complex of presheaves with terms of the form

$$
\underline{C}_{n}(F)(S)=F\left(S \times \Delta^{n}\right)
$$

and differentials given by alternated sums of homomorphisms induced by face maps in $\Delta^{\bullet}$. It is called the singular simplicial complex of $F$. As was shown in $[15$, Prop. 3.6] the cohomology presheaves

$$
\underline{h}_{i}(F)=\underline{H}^{-i}\left(\underline{C}_{*}(F)\right)
$$

are homotopy invariant for any $F$.

For any smooth scheme $X$ over $k$ we defined in [16] a presheaf $L(X)$ on the category $S m / k$ such that for any smooth connected scheme $U$ over $k$ $L(X)(U)$ is the free abelian group generated by closed irreducible subsets of $X \times U$ which are finite and surjective over $U$. The presheaves $L(X)$ have canonical structure of presheaves with transfers (see [16]).

Consider the presheaf $L\left(\left(\mathbf{A}^{1}-\{0\}\right)^{n}\right)$ and let $F_{n}$ be the sum of images of homomorphisms

$$
L\left(\left(\mathbf{A}^{1}-\{0\}\right)^{n-1}\right) \rightarrow L\left(\left(\mathbf{A}^{1}-\{0\}\right)^{n}\right)
$$

induced by embeddings of the form

$$
\left(x_{1}, \ldots, x_{n-1}\right) \mapsto\left(x_{1}, \ldots, 1, \ldots, x_{n-1}\right) .
$$

One can verify easily that $F_{n}$ is a direct summand of $L\left(\left(\mathbf{A}^{1}-\{0\}\right)^{n}\right)$ 
Definition 2.1 The motivic complex $\mathbf{Z}(n)$ of weight $n$ on $S m / k$ is the complex $\underline{C}_{*}\left(L\left(\left(\mathbf{A}^{1}-\{0\}\right)^{n}\right) / F_{n}\right)$ shifted by $-n$.

Note that $\mathbf{Z}(n)$ is a complex of presheaves with transfers with homotopy invariant cohomology presheaves.

For a smooth scheme $X$ over $k$ we define its motivic cohomology groups $H_{\mathcal{M}}^{i}(X, \mathbf{Z}(j))$ as hypercohomology $\mathbf{H}_{Z a r}^{i}(X, \mathbf{Z}(j))$. We will often abbreviate this notation writing $H^{i}\left(-, \mathbf{Z}(n)\right.$ instead of $H_{\mathcal{M}}^{i}\left(-, \mathbf{Z}(n)\right.$ and $H^{i}(F, \mathbf{Z}(n))$ instead of $H^{i}(\operatorname{Spec}(F), \mathbf{Z}(n))$ for a field $F$. We use the same abbreviations for Zariski hypercohomology with coefficients in other complexes of sheaves.

In [16] we constructed a certain tensor triangulated category $D M_{g m}^{e f f}(k)$, a functor $M: S m / k \rightarrow D M_{g m}^{e f f}(k)$ and an object $\mathbf{Z}(1)$ in $D M_{g m}^{e f f}(k)$ such that one has:

$$
H^{i}(X, \mathbf{Z}(n))=H_{D m_{D M}}(M(X), \mathbf{Z}(n)[i])
$$

where $\mathbf{Z}(n)=\mathbf{Z}(1)^{\otimes n}$. This description of motivic cohomology allows us to deduce some of their properties from the corresponding general properties of the category $D M_{g m}^{e f f}(k)$ and functor $M$.

Motivic cohomology are "known" for weights 0 and 1 . Namely one has:

Proposition 2.2 1. The complex $\mathbf{Z}(0)$ is canonically quasi-isomorphic to the constant sheaf $\mathbf{Z}$.

2. The complex $\mathbf{Z}(1)$ is canonically quasi-isomorphic to the sheaf $\mathbf{G}_{m}$ placed in cohomological degree -1 .

In particular for a smooth connected scheme $X$ one has:

$$
\begin{gathered}
H^{i}(X, \mathbf{Z}(0))=\left\{\begin{array}{lll}
\mathbf{Z} & \text { for } i=0 \\
0 & \text { for } i \neq 0
\end{array}\right. \\
H^{i}(X, \mathbf{Z}(1))= \begin{cases}\mathcal{O}^{*}(X) & \text { for } i=1 \\
\operatorname{Pic}(X) & \text { for } i=2 \\
0 & \text { for } i \neq 1,2\end{cases}
\end{gathered}
$$

It follows immediately from the definition that motivic cohomology have Mayer-Vietoris property for Zariski open coverings. Another type of long exact sequences for motivic cohomology is given by the following proposition. 
Proposition 2.3 Let $X$ be a smooth scheme over $k, Z \subset X$ be a smooth closed subscheme of $X$ and $p: X_{Z} \rightarrow X$ be the blowup of $Z$ in $X$. Then there are canonical long exact sequences of the form:

$$
\begin{aligned}
\ldots & \rightarrow H^{i}(X, \mathbf{Z}(j)) \rightarrow H^{i}\left(X_{Z}, \mathbf{Z}(j)\right) \oplus H^{i}(Z, \mathbf{Z}(j)) \rightarrow \\
& \rightarrow H^{i}\left(p^{-1}(Z), \mathbf{Z}(j)\right) \rightarrow H^{i+1}(X, \mathbf{Z}(j)) \rightarrow \ldots
\end{aligned}
$$

Proof: It follows from the existence of a distinguished triangle of the form

$$
M\left(p^{-1}(Z)\right) \rightarrow M\left(X_{Z}\right) \oplus M(Z) \rightarrow M(X) \rightarrow M\left(p^{-1}(Z)\right)[1]
$$

in the category $D M_{g m}^{e f f}(k)$. See [16, Prop. 3.5.2].

Remark: It can be shown that the long exact sequence from Proposition 2.3 has a canonical splitting and if $k$ admits resolution of singularities we have:

$$
H^{i}\left(X_{Z}, \mathbf{Z}(n)\right)=H^{i}(X, \mathbf{Z}(n)) \oplus\left(\bigoplus_{j=0}^{c-1} H^{i-2 j}(Z, \mathbf{Z}(n-j))\right) .
$$

Since motivic cohomology are defined as hypercohomology groups with coefficients in certain complexes of sheaves one can also define for any closed subscheme $Z$ in a smooth scheme $X$ motivic cohomology of $X$ with supports in $Z$. We denote them by $H_{Z}^{*}(X, \mathbf{Z}(n))$. As for any hypercohomology with supports we have canonical long exact sequences of the form:

$$
\begin{aligned}
\ldots \rightarrow H_{Z}^{i}(X, \mathbf{Z}(n)) & \rightarrow H^{i}(X, \mathbf{Z}(n)) \rightarrow H^{i}(X-Z, \mathbf{Z}(n)) \rightarrow \\
& \rightarrow H_{Z}^{i+1}(X, \mathbf{Z}(n)) \rightarrow \ldots
\end{aligned}
$$

Proposition 2.4 Let $k$ be a field which admits resolution of singularities, $X$ be a smooth scheme over $k$ and $Z$ be a smooth closed subscheme of $X$ of pure codimension $c$. Then there are canonical isomorphisms

$$
H_{Z}^{i}(X, \mathbf{Z}(n))=H^{i-2 c}(Z, \mathbf{Z}(n-c)) .
$$


Proof: By [16, Prop. 3.5.4] for any field $k$ there is a distinguished triangle in $D M_{g m}^{e f f}(k)$ of the form:

$$
M(X-Z) \rightarrow M(X) \rightarrow M(Z)(c)[2 c] \rightarrow M(X-Z)[1]
$$

such that we have

$$
H_{Z}^{i}(X, \mathbf{Z}(n))=\operatorname{Hom}_{D M}(M(Z)(c)[2 c], \mathbf{Z}(n)[i]) .
$$

Our proposition follows now from the quasi-invertibility of Tate object in $D M_{g m}^{e f f}(k)$ for fields $k$ which admit resolution of singularities (see [16, Th. 4.3.1].

As a corollary we obtain the Gysin long exact sequences in motivic cohomology. Namely for any $k, X$ and $Z$ as in Proposition 2.4 we have long exact sequences of the form:

$$
\begin{aligned}
& \ldots \rightarrow H^{i-2 c}(Z, \mathbf{Z}(n-c)) \rightarrow H^{i}(X, \mathbf{Z}(n)) \rightarrow \\
\rightarrow & H^{i}(X-Z, \mathbf{Z}(n)) \rightarrow H^{i-2 c+1}(Z, \mathbf{Z}(n-c)) \rightarrow \ldots
\end{aligned}
$$

Finally we would like to mention here the Projective Bundle theorem for motivic cohomology which allows in particular to construct using the standard Grothendieck's approach the characteristic classes with values in $H^{*}(-, \mathbf{Z}(*))$.

Proposition 2.5 Let $k$ be a field which admits resolution of singularities, $X$ be a smooth scheme over $k$ and $\mathcal{E}$ be a vector bundle on $X$ of pure dimension $d$. denote by $\mathbf{P}(\mathcal{E}) \rightarrow X$ the associated projective bundle. Then there are canonical isomorphisms:

$$
H^{i}(\mathbf{P}(\mathcal{E}), \mathbf{Z}(n))=\bigoplus_{j=0}^{d-1} H^{i-2 j}(X, \mathbf{Z}(n-j)) .
$$

Proof: It follows from the projective bundle theorem in $D M_{g m}^{e f f}(k)$ which holds for any field $k$ ([16, Prop. 3.5.1]) and quasi-invertibility of the Tate object for fields which admit resolution of singularities ([16, Th. 4.3.1]).

As conjectured by A. Beilinson and S. Lichtenbaum motivic complexes (and afortiory, motivic cohomology) should satisfy several good properties 
many of which we are now able to prove (at least in the case of fields which admit resolution of singularities). Among the ones which we do not know at the moment are the Beilinson-Lichtenbaum conjecture which is discused in Section 5 and the following conjecture known as the Beilinson-Soule vanishing conjecture.

Conjecture 2.6 For any smooth scheme $X$ over $k$, any $n$ and any $i<0$ one has

$$
H^{i}(X, \mathbf{Z}(n))=0 \text {. }
$$

By Proposition 2.2 this conjecture holds for $n \leq 1$. As a corollary of our main theorem (Theorem 5.9) we will show that it also holds for $n=2$ if one considers finite coefficients of torsion prime to $\operatorname{char}(k)$ and for $n=3,4$ if one considers $\mathbf{Z} / 2$-coefficients and $k$ is a field of characteristic $\neq 2$.

In this paper we will be mostly interested in motivic cohomology with finite coefficients. Define for any abelian group $A$ a complex of sheaves $A(n)$ as the tensor product $\mathbf{Z}(n) \otimes \underline{A}$ where $\underline{A}$ is the constant sheaf associated with $A$. Note also that for any presheaf with transfers $F$ and a constant sheaf $A$ the sheaf $F \otimes A$ has a canonical structure of a presheaf with transfers. The groups $\mathbf{Z}(n)(X)$ are torsion free and therefore the naive tensor product $\mathbf{Z}(n) \otimes A$ coicides with the tensor product $\mathbf{Z}(n) \stackrel{L}{\otimes} A$ in the derived category of sheaves. In particular, for any short exact sequence of abelian groups

$$
0 \rightarrow A_{1} \rightarrow A_{2} \rightarrow A_{2} \rightarrow 0
$$

we have a distinguished triangle of complexes of the form

$$
A_{1}(n) \rightarrow A_{2}(n) \rightarrow A_{3}(n) \rightarrow A_{1}(n)[1]
$$

and therefore a long exact sequence of motivic cohomology groups

$$
\begin{aligned}
& \ldots \rightarrow H^{i}\left(X, A_{1}(n)\right) \rightarrow H^{i}\left(X, A_{2}(n)\right) \rightarrow \\
& \rightarrow H^{i}\left(X, A_{3}(n)\right) \rightarrow H^{i+1}\left(X, A_{1}(n)\right) \rightarrow \ldots
\end{aligned}
$$

For the proof of our main theorem (Theorem 5.9) we will need to use motivic cohomology of non-smooth schemes. Let $S c h / k$ be the category of schemes of finite type over $k$. There is an obvious morphism of sites

$$
\pi:(S c h / k)_{c d h} \rightarrow(S m / k)_{Z a r}
$$


where $c d h$ denotes the cdh-topology introduced in [14],[5]. By abuse of notations we denote for a sheaf (or complex of sheaves) $F$ on $(S m / k)_{Z a r}$ by $F_{c d h}$ the complex $\pi^{*}(F)$ on $(S c h / k)_{c d h}$. As was shown in [5, Lemma 3.6] the functor $\pi^{*}$ is exact if $k$ admits resolution of singularities.

For any scheme of finite type $X$ over a field $k$ which admits resolution of singularities we set:

$$
H^{i}(X, \mathbf{Z}(n))=\mathbf{H}_{c d h}^{i}\left(X, \mathbf{Z}(n)_{c d h}\right)
$$

By [5, Th. 5.5(1)] this definition agrees for smooth schemes with the one given above. It also agrees in this case with the definition of motivic cohomology given in [5, Def. 9.2]. More precisely it is easy to show using Mayer-Vietoris property of algebraic singular homology proven in [15] that for any field $k$ the complex $\mathbf{Z}(n)[2 n]$ is canonically quasi-isomorphic in Zariski topology on the category $S m / k$ of smooth schemes over $k$ to the complex $\underline{C}_{*}\left(c_{e q u i}\left(\mathbf{P}^{n}, 0\right)\right) / \underline{C}_{*}\left(c_{\text {equi }}\left(\mathbf{P}^{n-1}, 0\right)\right)$. The inverse image of the later complex to $(S c h / k)_{c d h}$ is canonically quasi-isomorphic for a field $k$ which admits resolution of singularities to $\underline{C}_{*}\left(z\left(\mathbf{A}^{n}, 0\right)\right)$ by the localization theorem which implies the comparison with the definition given in [5].

The following proposition is a direct corollary of the definition of cdhtopology.

Proposition 2.7 Let $X$ be a scheme of finite type over $k, Z$ be a closed subscheme in $X$ and $p: \tilde{X} \rightarrow X$ be a proper morphism such that $p^{-1}(X-$ $Z) \rightarrow X-Z$ is an isomorphism. Then there is a canonical long exact sequence of the form:

$$
\begin{aligned}
\ldots \rightarrow & H^{i}(X, \mathbf{Z} / l(n)) \rightarrow H^{i}(\tilde{X}, \mathbf{Z} / l(n)) \oplus H^{i}(Z, B / l(n)) \rightarrow \\
& \rightarrow H^{i}\left(p^{-1}(Z), \mathbf{Z} / l(n)\right) \rightarrow H^{i+1}(X, \mathbf{Z} / l(n)) \rightarrow \ldots
\end{aligned}
$$

Corollary 2.8 Let $X$ be a scheme of finite type over $k$ and $X=Y_{1} \cup Y_{2}$ be a closed covering of $X$. Then there are canonical long exact sequences of the form

$$
\begin{aligned}
\ldots \rightarrow & H^{i}(X, \mathbf{Z} / l(n)) \rightarrow H^{i}\left(Y_{1}, \mathbf{Z} / l(n)\right) \oplus H^{i}\left(Y_{2}, \mathbf{Z} / l(n)\right) \rightarrow \\
& \rightarrow H^{i}\left(Y_{1} \cap Y_{2}, \mathbf{Z} / l(n)\right) \rightarrow H^{i+1}(X, \mathbf{Z} / l(n)) \rightarrow \ldots
\end{aligned}
$$


Remark: Proposition 2.5 can be immediately generalized to non-smooth schemes if $k$ admits resolution of singularities. However, the isomorphisms of Proposition 2.2 do not hold in this generality. For example, for any scheme $X$ the group $H^{1}(X, \mathbf{Z}(1))$ is canonically isomorphic to $\mathcal{O}^{*}\left(X_{\text {red }}\right)$ where $X_{\text {red }}$ is the maximal reduced subscheme of $X$. The group $H^{2}(X, \mathbf{Z}(1))$ being always isomorphic to $H_{c d h}^{1}\left(X, \mathcal{O}_{c d h}^{*}\right)$ is not necessarily isomorphic to the Picard group even for normal schemes $X$.

Let $S$ be a scheme over $k$ and $p: X \rightarrow S$ be a scheme of finite type over $S$. In [14] we defined an abelian group $c(X / S, 0)$ of proper relative cycles of relative dimension 0 on $X$ over $S$. Similarly, one can define an abelian group $c(X / S, 0, \mathbf{Z} / l)$ of proper relative cycles of relative dimension 0 on $X$ over $S$ with $\mathbf{Z} / l$-coefficients. These groups are covariantly functorial with respect to morphisms of schemes of finite type over $S$.

They also form a presheaf on the category of schemes over $S$, i.e. for a morphism $g: S^{\prime} \rightarrow S$ we have canonical homomorphisms

$$
\begin{aligned}
\operatorname{cycl}(g): c(X / S, 0) & \rightarrow c\left(X \times{ }_{S} S^{\prime} / S^{\prime}, 0\right) \\
\operatorname{cycl}(g): c(X / S, 0, \mathbf{Z} / l) & \rightarrow c\left(X \times{ }_{S} S^{\prime} / S^{\prime}, 0, \mathbf{Z} / l\right)
\end{aligned}
$$

We need the following result.

Proposition 2.9 Let $S$ be a scheme over $k$ and $X \rightarrow S$ be a scheme of finite type over $S$. Then there are canonical pairings:

$$
\begin{gathered}
c(X / S, 0) \otimes H^{i}(X, \mathbf{Z}(n)) \rightarrow H^{i}(S, \mathbf{Z}(n)) \\
c(X / S, 0, \mathbf{Z} / l) \otimes H^{i}(X, \mathbf{Z} / l(n)) \rightarrow H^{i}(S, \mathbf{Z} / l(n))
\end{gathered}
$$

such that:

1. For a morphism $f: X_{1} \rightarrow X_{2}$ over $S$ and elements $\mathcal{Z}$ in $c\left(X_{1} / S, 0\right)$ (resp. in $c\left(X_{1} / S, 0, \mathbf{Z} / l\right)$ ) and $\alpha$ in $H^{i}\left(X_{2}, \mathbf{Z}(n)\right)$

(resp. in $H^{i}\left(X_{2}, \mathbf{Z} / l(n)\right)$ one has

$$
\left(\mathcal{Z}, f^{*}(\alpha)\right)=\left(f_{*}(\mathcal{Z}, \alpha)\right) .
$$

2. For a morphism $g: S^{\prime} \rightarrow S$ and elements $\mathcal{Z}$ in $c(X / S, 0)$ (resp. in $c(X / S, 0, \mathbf{Z} / l))$ and $\alpha$ in $H^{i}(X, \mathbf{Z}(n))$ (resp. in $H^{i}(X, \mathbf{Z} / l(n))$ one has

$$
g^{*}(\mathcal{Z}, \alpha)=\left(\operatorname{cycl}(g)(\mathcal{Z}), p r^{*}(\alpha)\right)
$$

where $\mathrm{pr}: X \times_{S} S^{\prime} \rightarrow X$ is the projection. 
Proof: We will only consider the integral case. The case of finite coefficients is similar. Since motivic cohomology may be described as morphisms in the category $D M_{g m}^{e f f}(k)$ it is sufficient to show that there is a homomorphism

$$
\omega: c(X / S, 0) \rightarrow \operatorname{Hom}_{D M}(M(S), M(X))
$$

such that the following conditions hold:

1. For a morphism $f: X_{1} \rightarrow X_{2}$ over $S$ and an element $\mathcal{Z}$ in $c\left(X_{1} / S, 0\right)$ one has

$$
f \circ \omega(\mathcal{Z})=\omega\left(f_{*}(\mathcal{Z})\right) .
$$

2. For a morphism $g: S^{\prime} \rightarrow S$ and an element $\mathcal{Z}$ in $c(X / S, 0)$ one has

$$
\omega(\mathcal{Z}) \circ f=\operatorname{pr} \circ \omega(\operatorname{cycl}(f)(\mathcal{Z}))
$$

where $\mathrm{pr}: X \times_{S} S^{\prime} \rightarrow X$ is the projection.

By definition of the functor $M: S c h / k \rightarrow D M_{g m}^{e f f}(k)$ there is a homomorphism

$$
c(X / \operatorname{Spec}(k), 0)(S) \rightarrow \operatorname{Hom}_{D M}(M(S), M(X)) .
$$

which is natural in both $X$ and $S$. It remains to construct a homomorphism

$$
\nu: c(X / S, 0) \rightarrow c(X / \operatorname{Spec}(k), 0)(S)=c\left(X \times_{\operatorname{Spec}(k)} S / S, 0\right)
$$

which is natural in the obvious sense with respect to morphisms in $X$ and $S$. For a cycle $\mathcal{Z}$ in $c(X / S, 0)$ we set:

$$
\nu(\mathcal{Z})=\operatorname{Cor}\left(\operatorname{pr}_{1}^{*}(\mathcal{Z}), \Delta\right)
$$

where $p r_{1}: S \times_{S p e c}(k) S$ is the projection, $\Delta \in c(S \times S / S, 0)$ is the diagonal cycle and $\operatorname{Cor}(-,-)$ is the correspondence homomorphism constructed in [14, 3.7]. The required naturality properties follow directly from the properties of correspondence homomorphisms proven in (loc.cit.)

Remark: If $k$ admits resolution of singularities one can construct similar pairings

$$
\begin{gathered}
c(X / S, d) \otimes H^{i}(X, \mathbf{Z}(n)) \rightarrow H^{i-2 d}(S, \mathbf{Z}(n-d)) \\
c(X / S, d, \mathbf{Z} / l) \otimes H^{i}(X, \mathbf{Z} / l(n)) \rightarrow H^{i-2 d}(S, \mathbf{Z} / l(n-d))
\end{gathered}
$$

for groups of proper relative cycles of relative dimension $d$. 


\section{Motivic cohomology and Milnor's K-theory.}

By definition of motivic cohomology we have

$$
H^{i}(\operatorname{Spec}(k), \mathbf{Z}(n))=0
$$

for $i>n$. In this section we will identify the first nontrivial motivic cohomology group of a field with Milnor's K-theory. Another proof of the same result based on the comparison of motivic cohomology with higher Chow groups can be found in [16].

We recall first the definition and some basic properties of the Milnor Ktheory. For a field $k$ denote by $\otimes^{*} k^{*}$ the tensor algebra of the abelian group $k^{*}$. Let $I^{*}$ be the graded ideal in $\otimes^{*} k^{*}$ generated by elements of the form $x \otimes y$ such that $x+y=1$. The quotient algebra $\otimes^{*} k^{*} / I^{*}$ is called the Milnor K-theory of $k$. The corresponding groups $\otimes^{n} k^{*} / I^{n}$ are denoted by $K_{n}^{M}(k)$. It can be shown that the multiplication in $K_{*}^{M}(k)$ is commutative in the graded sense, i.e. for $\phi \in K_{i}^{M}(k)$ and $\psi \in K_{j}^{M}(k)$ one has

$$
\phi \psi=(-1)^{i j} \psi \phi
$$

Clearly, Milnor's K-groups are contravariantly functorial with respect to arbitrary morphisms of the spectrums of fields. Another important property of Milnor's K-theory is the existence of transfers for finite field extensions.

Proposition 3.1 For any finite field extension $E / k$ there are well defined homomorphisms $N_{E / k, n}: K_{n}^{M}(E) \rightarrow K_{n}^{M}(k)$ satisfying the following conditions:

1. $N_{E / k, 0}: \mathbf{Z} \rightarrow \mathbf{Z}$ is the multiplication by $\operatorname{deg}(E / k)$ and $N_{E / k, 1}: E^{*} \rightarrow k^{*}$ is the usual norm homomorphism.

2. For an element $\phi \in K_{i}^{M}(E)$ and an element $\psi \in K_{j}^{M}(k)$ one has:

$$
N_{E / k, i+j}\left(\phi \otimes \psi_{E}\right)=N_{E / k, j}(\phi) \otimes \psi
$$

where $\psi_{E}$ is the image of $\psi$ in $K_{j}^{M}(E)$.

We are ready now to prove the following proposition relating Milnor's K-groups to motivic cohomology. 
Proposition 3.2 Let $k$ be a field. Then there are canonical isomorphisms

$$
H^{n}(\operatorname{Spec}(k), \mathbf{Z}(n))=K_{n}^{M}(k)
$$

compatible with transfers and multiplications in Milnor's K-theory and motivic cohomology respectively.

Proof: The case $n=1$ of this proposition is well-known and easy (cf. Proposition 2.2). It can be settled also following the lines of the proof below. To prove the general case denote temporarily the sheaf $L\left(\left(\mathbf{A}^{1}-\{0\}\right)^{n}\right) / F_{n}$ by $L_{n}$. For any irreducible $X \in S m / k$ the group $\Gamma\left(X, L_{n}\right)$ is a free abelian group generated by closed integral subschemes $Z \subset X \times\left(\mathbf{A}^{1}-\{0\}\right)^{n}$ which are finite and surjective over $X$ modulo a subgroup generated by those $Z$ which lie in $X \times\left(\mathbf{A}^{1}-\{0\}\right)^{i-1} \times 1 \times\left(\mathbf{A}^{1}-\{0\}\right)^{n-i}$ for some $i(1 \leq i \leq n)$. According to definitions the group $H^{n}(\operatorname{Spec}(k), \mathbf{Z}(n))$ coincides with the cokernel of the homomorphism

$$
L_{n}\left(\mathbf{A}^{1}\right) \stackrel{\partial_{0}-\partial_{1}}{\longrightarrow} L_{n}(\operatorname{Spec}(k))
$$

where $\partial_{0}$ and $\partial_{1}$ are homomorphisms induced by closed points

$$
0,1: \operatorname{Spec}(k) \hookrightarrow \mathbf{A}^{1} .
$$

We'll employ a special notation $H^{n}(k)$ for this cokernel. Observe that $H^{n}$ is a covariant functor oh the category of all fields. Furthermore product of cycles defines a pairing $H^{n}(k) \otimes H^{m}(k) \rightarrow H^{n+m}(k)$ which makes $\bigsqcup_{i=0}^{\infty} H^{i}(k)$ into a graded associative ring. Finally taking direct images of cycles we get a transfer homomorphism $\operatorname{Tr}_{k^{\prime} / k}: H^{n}\left(k^{\prime}\right) \rightarrow H^{n}(k)$ defined for any finite field extension $k^{\prime} / k$. The properties of these transfer maps summarized below follow immediately from the corresponding properties of the direct image homomorphism for cycles

Lemma 3.3 Let $k^{\prime} / k$ be a finite field extension and let further $K / k$ be a normal field extension such that $\operatorname{Hom}_{k}\left(k^{\prime}, K\right) \neq \emptyset$. For any $x \in H^{n}\left(k^{\prime}\right)$ and $y \in H^{m}(k)$ we have:

$$
\begin{aligned}
& \text { 1. } \operatorname{Tr}_{k^{\prime} / k}\left(y_{k^{\prime}} \cdot x\right)=y \cdot \operatorname{Tr}_{k^{\prime} / k}(x), \operatorname{Tr}_{k^{\prime} / k}\left(x \cdot y_{k^{\prime}}\right)=\operatorname{Tr}_{k^{\prime} / k}(x) \cdot y \\
& \text { 2. }\left(\operatorname{Tr}_{k^{\prime} / k}(x)\right)_{K}=\left[k^{\prime}: k\right]_{i} \cdot \sum_{j \in \operatorname{Hom}_{k}\left(k^{\prime}, K\right)}\left(j_{*}(x)\right)
\end{aligned}
$$


Note that every $k$-rational point of $\left(\mathbf{A}^{1} A^{1}-\{0\}\right)^{n}$ defines an element in $L_{n}(\operatorname{Spec}(k))$. In this way we get a canonical homomorphism $\mathbf{Z}\left[\left(k^{*}\right)^{n}\right] \rightarrow$ $H^{n}(k)$. We'll denote the image of the point $\left(a_{1}, \ldots, a_{n}\right)$ under this homomorphism by $\left[a_{1}, \ldots, a_{n}\right]$. The following relation is straightforward from the definition of $H^{n}$

$$
\left[a_{1}, \ldots, 1, \ldots, a_{n}\right]=0
$$

Note also the following relation which is obvious from the definition of products

$$
\left[a_{1}, \ldots, a_{k}\right] \cdot\left[a_{k+1}, \ldots, a_{n}\right]=\left[a_{1}, \ldots, a_{n}\right]
$$

Let $a, b \in k^{*}-\{1\}$ be any two elements. Consider the closed subscheme $Y \subset \mathbf{A}^{1} \times\left(\mathbf{A}^{1}-\{0\}\right)$, given by the equation

$$
X^{2}-(t \cdot(a+b)+(1-t) \cdot(1+a b)) X+a b=0
$$

(here $t$ is the coordinate in $\mathbf{A}^{1}$ and $X$ is the coordinate in $\mathbf{A}^{1}-\{0\}$ ). One checks immediately that the projection $p_{2}: Y \rightarrow \mathbf{A}^{1}-\{0\}$ is an isomorphism so that in particular $Y$ is integral. Moreover it's clear that $Y$ is finite and surjective over $\mathbf{A}^{1}$ and hence defines an element $y \in L_{1}\left(\mathbf{A}^{1}\right)$. Since $\partial_{0}(y)=$ $[a b]$ and $\partial_{1}(y)=[a]+[b]$ we conclude that $[a b]=[a]+[b]$.

Next consider the closed embedding $Y \hookrightarrow \mathbf{A}^{1} \times\left(\mathbf{A}^{1}-\{0\}\right)^{2}$ obtained by means of the diagonal embedding $\mathbf{A}^{1}-\{0\} \hookrightarrow\left(\mathbf{A}^{1}-\{0\}\right)^{2}$. Using the same argument as above we conclude that $[a b, a b]=[a, a]+[b, b]$. This relation together with bimultiplicativity of the symbol $[-,-]$ imply immediately that $2 \cdot[a,-a]=0 \quad \forall a \in k^{*}$. The following lemma shows that in fact $[a,-a]=$ $0 \quad \forall a \in k^{*}$

Lemma 3.4 Assume that there exists an integer $N>0$ such that $N \cdot[a, 1-$ $a]=0$ for any field $k$ and any element $a \in k^{*}-\{1\}$ (resp. $N \cdot[a,-a]=0$ for any $k$ and any $a \in k^{*}$ ). Then $[a, 1-a]=0 \quad \forall k \quad \forall a \in k^{*}-\{0\}$ (resp. $\left.[a,-a]=0 \quad \forall k \quad \forall a \in k^{*}\right)$.

Proof: The proof is the same for both cases so we'll consider only the first one. It suffices to show that if all symbols of the form $[a, 1-a]$ are killed by an integer $N=M p$, where $p$ is a prime then all such symbols are killed by $M$ already. The case $a \in\left(k^{*}\right)^{p}$ is easy so we'll assume that $a$ is not $p$-th power. Set $\alpha=a^{1 / p}, k^{\prime}=k(\alpha)$. According to our assumptions $0=M p \cdot[\alpha, 1-\alpha]=$ 
$M \cdot[a, 1-\alpha]$. Applying to this equation the homomorphism $\operatorname{Tr}_{k^{\prime} / k}$ and using Lemma 3.3 we get:

$$
\begin{gathered}
0=\operatorname{Tr}_{k^{\prime} / k}(M \cdot[a, 1-\alpha])=M \cdot[a] \cdot \operatorname{Tr}_{k^{\prime} / k}([1-\alpha])=M \cdot[a] \cdot\left[N_{k^{\prime} / k}(1-\alpha)\right] \\
=M \cdot[a, 1-a]
\end{gathered}
$$

The relation $[a,-a]=0$ implies immediately that $[a, 1-a]+\left[a^{-1}, 1-a^{-1}\right]=$ $0 \forall a \in k^{*}-\{1\}$. Assume now that $a \in k^{*}$ is an element such that $a^{6} \neq 1$. Consider a closed subscheme $Z \subset \mathbf{A}^{1} \times\left(\mathbf{A}^{1}-\{0,1\}\right)$ given by the equation

$$
X^{3}-t \cdot\left(a^{3}+1\right) \cdot X^{2}+t \cdot\left(a^{3}+1\right) \cdot X-a^{3}=0
$$

Once again one checks immediately that the projection $p_{2}: Z \rightarrow \mathbf{A}^{1}-\{0,1\}$ is an isomorphism, so that $Z$ is, in particular, integral. The fiber of $Z$ over $0 \in \mathbf{A}^{1}$ consists of all cubical roots of $y^{3}$ and the fiber of $Z$ over $1 \in \mathbf{A}^{1}$ consists of $y^{3}$ and two roots $x_{1}, x_{2}$ of the equation $X^{2}-X+1=0$. We embed $Z$ into $\mathbf{A}^{1} \times\left(\mathbf{A}^{1}-\{0\}\right)^{2}$ using the embedding $\mathbf{A}^{1}-\{0,1\} \hookrightarrow\left(\mathbf{A}^{1}-\{0\}\right)^{2} \quad x \mapsto$ $(x, 1-x)$. Assume for the moment that $k$ contains all roots of the equation $X^{6}-1=0$. In this case $\partial_{0}(Z)=[a, 1-a]+[\xi a, 1-\xi a]+\left[\xi^{2} a, 1-\xi^{2} a\right]$, where $\xi$ is the generator of the group $\mu_{3}(\xi=1$ if $\operatorname{char}(k)=3)$. At the same time $\partial_{1}(Z)=\left[a^{3}, 1-a^{3}\right]+\left[x_{1}, 1-x_{1}\right]+\left[x_{2}, 1-x_{2}\right]=\left[a^{3}, 1-a^{3}\right]$. This gives us the relation $2\left[a^{3}, 1-a^{3}\right]=0$. Using once again the transfer argument we conclude further that $6[a, 1-a]=0$ for any $a \in k^{*}$.Lemma 3.4 shows now that $[a, 1-a]=0 \quad \forall a \in k^{*}-\{1\}$.

The above relations show that we have a canonical homomorphism $\lambda$ : $K_{n}^{M}(k) \rightarrow H^{n}(k)$ sending $\left\{a_{1}, \ldots, a_{n}\right\}$ to $\left[a_{1}, \ldots, a_{n}\right]$. Next we verify that this homomorphism is compatible with transfers. Assume first that any finite extension of $k$ is $p$-primary where $p$ is a certain prime. In this case any finite extension of $k$ may be decomposed into a tower of normal extensions of degree $p$. So it suffices to consider the case when $k^{\prime} / k$ has degree exactly $p$. A theorem due to Bass and Tate [1] shows that in this case $K_{n}^{M}\left(k^{\prime}\right)$ is generated by $K_{n-1}^{M}(k) \cdot K_{1}^{M}\left(k^{\prime}\right)$. Lemma 3.3 and the projection formula in Milnor K-theory allow to reduce the general case to the case $n=1$ which is trivial. In general for any prime integer $p$ we may find an algebraic extension $K / k$ of degree prime to $p$ such that any finite extension of $K$ is $p$-primary. Lemma 3.3 and the corresponding result in Milnor K-theory together with the 
case settled above show that for any $v \in K_{n}^{M}\left(k^{\prime}\right)$ the element $\lambda\left(\operatorname{Tr}_{k^{\prime} / k}(v)-\right.$ $\operatorname{Tr}_{k^{\prime} / k}\left(\lambda(v)\right.$ dies in $H^{n}(K)$ and hence is killed by an integer prime to $p$. Since $p$ was arbitrary we conclude that $\lambda\left(\operatorname{Tr}_{k^{\prime} / k}(v)=\operatorname{Tr}_{k^{\prime} / k}(\lambda(v))\right.$.

The definition of $H^{n}(k)$ shows that this group is generated by transfers of rational points. Since each element of this type lies in the image of $K_{n}^{M}(k)$ we conclude that the homomorphism $\lambda$ is surjective.

To finish the proof we define a homomorphism $H^{n}(k) \rightarrow K_{n}^{M}(k)$ inverse to $\lambda$. Let $v \in\left(\mathbf{A}_{k}^{1}-\{0\}\right)^{n}$ be any closed point. The residue field $k(v)$ is a finite extension of $k$ and we set $\theta(v)=\operatorname{Tr}_{k(v) / k}\left(\left\{X_{1}(v), \ldots, X_{n}(v)\right\}\right) \in K_{n}^{M}(k)$. The usual argument involving the Weil reciprocity formula (see [9]) shows that the homomorphism $\theta: L_{n}\left(\operatorname{Spec}(k) \rightarrow K_{n}^{M}(k)\right.$ kills the image of $\partial_{0}-\partial_{1}$ and thus defines a homomorphism $\theta: H^{n}(k) \rightarrow K_{n}^{M}(k)$ inverse to $\lambda$.

Corollary 3.5 For any field $k$ and any integer $m>0$ there is a canonical isomorphism

$$
H^{n}(\operatorname{Spec}(k), \mathbf{Z} / l(n))=K_{n}^{M}(k) / l .
$$

Proof: It follows from Proposition 3.2 by the long exact sequence which relates motivic cohomology with finite and integral coefficients and the fact that $H^{n+1}(\operatorname{Spec}(k), \mathbf{Z}(n))=0$.

\section{Bloch-Kato conjecture.}

In this section we recall the Bloch-Kato conjecture relating Milnor's K-theory to etale cohomology in the case of fields. In the next section we will discuss a stronger (in view of Corollary 3.5) conjecture due to A. Beilinson and S. Lichtenbaum which describes all motivic cohomology with finite coefficients in terms of etale cohomology.

From now on we fix a prime $l$ which we always assume not to be equal to the characteristic of our base field $k$.

We have $K_{1}^{M}(k)=k^{*}$ and the Kummer exact sequence gives us a homomorphism

$$
\eta_{1}: K_{1}^{M}(k) \rightarrow H_{e t}^{1}\left(k, \mu_{l}\right)
$$

where $\mu_{l}$ is the sheaf of l-th roots of unit. Using multiplicative structure in etale cohomology we further get a homomorphism:

$$
\eta_{1}^{\otimes n}:\left(k^{*}\right)^{\otimes n} \rightarrow H_{e t}^{n}\left(k, \mu_{l}^{\otimes n}\right)
$$


(where $\mu_{l}^{\otimes n}$ is the $\mathrm{n}$-th tensor power of $\mu_{l}$ over $\mathbf{Z} / l$ ).

The following result is well known.

Proposition 4.1 The homomorphism $\eta_{1}^{\otimes n}$ factors through a homomorphism

$$
\eta_{n}: K_{n}^{M}(k) \rightarrow H_{e t}^{n}\left(k, \mu_{l}^{\otimes n}\right)
$$

which is compatible with transfers.

Proof: To construct $\eta_{n}$ it is sufficient by definition of Milnor's K-theory to show that for an element $x \neq 1$ of $k^{*}$ we have $\eta_{1}^{\otimes 2}(x \otimes(1-x))=0$ in $H_{e t}^{2}\left(k, \mu_{l}^{\otimes 2}\right)$. Consider the cyclic extension $E=k\left(x^{1 / l}\right)$ of $k$.

We have:

$$
\begin{gathered}
\eta_{1}^{\otimes 2}(x \otimes(1-x))=\eta_{1}(x) \otimes \eta_{1}(1-x)=\operatorname{Tr}_{E / k}\left(\eta_{1}(x) \otimes \eta_{1}\left(1-x^{1 / l}\right)\right)= \\
=l T r_{E / k}\left(\eta_{1}\left(x^{1 / l}\right) \otimes \eta_{1}\left(1-x^{1 / l}\right)\right)=0 .
\end{gathered}
$$

To verify that $\eta_{n}$ is compatible with transfers it is sufficient to show that it is true in the case of a cyclic extension $E / k$ of a prime degree $p$. By [1] any element of $K_{n}^{M}(E)$ is representable by a sum of symbols of the form $\left(x_{1}, \ldots, x_{n-1}, y\right)$ where $x_{1}, \ldots, x_{n-1} \in k^{*}$ and

$$
\operatorname{Tr}_{E / k}\left(x_{1}, \ldots, x_{n-1}, y\right)=\left(x_{1}, \ldots, x_{n-1}, N_{E / k}(y)\right)
$$

where $N_{E / k}: E^{*} \rightarrow k^{*}$ is the usual norm homomorphism. It implies immediately that the homomorphisms $\eta_{n}$ are compatible with transfers.

We state Bloch-Kato conjecture (over $k$ with respect to the prime $l$ ) in weight $n$ in two forms a strong and a weak one.

Weak form. For any field $F$ over $k$ the homomorphism $\eta_{n}$ is surjective.

Strong form. For any field $F$ over $k$ the homomorphism $\eta_{n}$ gives an isomorphism

$$
K_{n}^{M}(F) / l \cong H_{e t}^{n}\left(F, \mu_{l}^{\otimes n}\right) .
$$

We will show below that these two forms of Bloch-Kato conjecture are in fact equivalent at least when $k$ admits resolution of singularities.

Everywhere below "Bloch-Kato conjecture" refers to the weak form unless the strong form is explicitly specified.

The following theorem summarizes the cases in which the strong form of Bloch-Kato conjecture is known today. 
Theorem 4.2 Let $k$ be a field and $l$ be an integer prime to char $(k)$. The homomorphism

$$
K_{n}^{M} / l(k) \rightarrow H_{e t}^{n}\left(k, \mu_{l}^{\otimes n}\right)
$$

is an isomorphism in the following cases:

1. For $n=0,1$ (it follows from the classical Theorem Hilbert 90).

2. For $n=2$ see [7], [11].

3. For $n=3, l=2$ see [8], [10]

4. For $n=4, l=2$ (M. Rost, unpublished).

5. For a field $k$ which contains an algebraically closed subfield $k_{0}$ which admits resolution of singularities if $n \geq \operatorname{deg}_{t r}\left(k / k_{0}\right)$ (it follows from results of [13]).

We will often use below the following two simple results.

Lemma 4.3 Let $k$ be a field such that Bloch-Kato conjecture (resp. the strong form of Bloch-Kato conjecture) holds over $k$ in weight $n$. Then it also holds over $k$ in all weights less than $n$.

Proof: It is sufficient to show that under our assumption the Bloch-Kato conjecture holds for weight $n-1$. Let $F$ be a field of finite type over $k$ and $F(t)$ be the field of rational functions of one variable over $F$. Homomorphisms $\eta_{n}$ and $\eta_{n-1}$ form a morphism of short exact sequences:

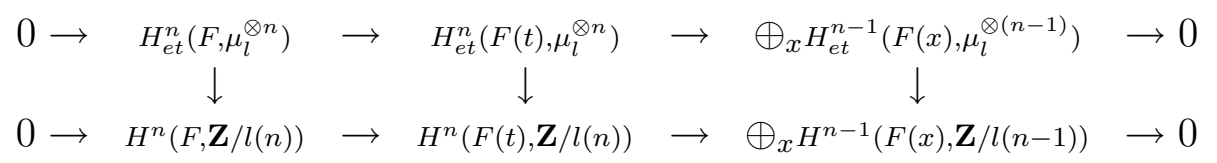

where $x$ runs through the set of closed points of $\mathbf{A}_{F}^{1}$ and $F(x)$ is the residue field of $x$. By our assumption the left hand side vertical arrow and the middle vertical arrow are surjections (resp. isomorphisms). Therefore so is the right hand side arrow.

Lemma 4.4 Let $k$ be a field and $k^{\prime}=k(\xi)$ where $\xi$ is a primitive l-th root of unit. Then Bloch-Kato conjecture (respectively the strong form of Bloch-Kato conjecture) holds in weight $n$ over $k$ if and only if it holds in weight $n$ over $k^{\prime}$. 
Proof: It follows immediately from the fact that $k^{\prime} / k$ is an extension of degree $l-1$ and that homomorphisms $\eta_{n}$ are compatible with transfers.

\section{$5 \quad$ The truncated etale cohomology and Beilinson-Lichtenbaum conjecture.}

Consider the complexes $B / l(n)$ of sheaves in Zariski topology on $S m / k$ of the form

$$
B / l(n)=\tau_{\leq n} \mathbf{R} \pi_{*}\left(\mu_{l}^{\otimes n}\right)
$$

where $\pi:(S m / k)_{e t} \rightarrow(S m / k)_{Z a r}$ is the obvious morphism of sites and $\tau_{\leq n}(K)$ for a complex of sheaves $K$ is the part of the canonical filtration on $K$ such that:

$$
\underline{H}^{i}\left(\tau_{\leq n}(K)\right)= \begin{cases}\underline{H}^{i}(K) & \text { for } \quad i \leq n \\ 0 & \text { for } i>n\end{cases}
$$

It follows immediately from this definition that for any $i$ we have canonical homomorphisms

$$
H^{i}(X, B / l(n)) \rightarrow H_{e t}^{i}\left(X, \mu_{l}^{\otimes n}\right)
$$

which are isomorphisms for $i \leq n$ and monomorphisms for $i=n+1$ (let us recall that the notation $H^{*}(-, B / l(n))$ stays for hypercohomology in Zariski topology).

Proposition $5.1 \mathrm{~B} / \mathrm{l}(n)$ is a complex of presheaves with transfers with homotopy invariant cohomology sheaves.

By Proposition 5.1 we may consider $B / l(n)$ as objects of the triangulated category $D M^{e f f}(k)$ and we have

$$
H^{i}(X, B / l(n))=\operatorname{Hom}_{D M}(M(X), B / l(n)[i]) .
$$

Using this description and results of [16] one can show easily that analogs of Propositions 2.3 and 2.5 hold for cohomology with coefficients in $B / l(n)$. In the proof of Theorem 5.9 we will use cohomology with coefficients in $B / l(n)$ for non smooth schemes defined in exactly the same way as we did for motivic cohomology at the end of Section 2, i.e. for a scheme of finite type $X$ over $k$ we define $H^{i}(X, B / l(n))$ as $H_{c d h}^{i}\left(X,(B / l(n))_{c d h}\right)$. These cohomology groups satisfy the the following analog of Proposition 2.7. 
Proposition 5.2 Let $X$ be a scheme of finite type over $k, Z$ be a closed subscheme in $X$ and $p: \tilde{X} \rightarrow X$ be a proper morphism such that $p^{-1}(X-$ $Z) \rightarrow X-Z$ is an isomorphism. Then there is a canonical long exact sequence of the form:

$$
\begin{aligned}
\ldots \rightarrow & H^{i}(X, B / l(n)) \rightarrow H^{i}(\tilde{X}, B / l(n)) \oplus H^{i}(Z, B / l(n)) \rightarrow \\
& \rightarrow H^{i}\left(p^{-1}(Z), B / l(n)\right) \rightarrow H^{i+1}(X, B / l(n)) \rightarrow \ldots
\end{aligned}
$$

The proper base change theorem for etale cohomology implies the following fact.

Proposition 5.3 Let $X$ be a scheme of finite type over a field $k$ which admits resolution of singularities. Then for any $i \in \mathbf{Z}$ there is a canonical morphism

$$
H^{i}(X, B / l(n)) \rightarrow H_{e t}^{i}(X, B / l(n))
$$

which is an isomorphism for $i \leq n$ and a monomorphisms for $i=n+1$.

The following proposition relates complexes $\mathbf{Z} / l(n)$ and $B / l(n)$.

Proposition 5.4 There is a canonical morphism

$$
\mathbf{Z} / l(n) \rightarrow B / l(n)
$$

in the derived category of homotopy invariant sheaves with transfers such that the corresponding homomorphism

$$
K_{n}^{M} / l=\underline{H}^{n}(\mathbf{Z} / l(n))(\operatorname{Spec}(k)) \rightarrow \underline{H}^{n}(B / l(n))(\operatorname{Spec}(k))=H_{e t}^{n}\left(k, \mu_{l}^{\otimes n}\right)
$$

coincides with the homomorphism $\eta_{n}$ constructed in Section 4.

Proposition 5.4 implies in particular that there are canonical homomorphisms

$$
H^{i}(X, \mathbf{Z} / l(n)) \rightarrow H^{i}(X, B / l(n))
$$

compatible with transfers.

We will need the analog of Proposition 2.4 which follows from the interpretation of cohomology with coefficients in $B / l(n)$ in terms of morphisms in $D M^{e f f}(k)$ in exactly the same way as the proof of Proposition 2.4. 
Proposition 5.5 Let $X$ be a smooth scheme over $k$ and $Z \subset X$ be a smooth closed subscheme of $X$ of pure codimension c. Then there are canonical isomorphisms

$$
H_{Z}^{i}(X, B / l(n)) \cong H^{i-2 c}(Z, B / l(n-c))
$$

compatible with the corresponding isomorphisms for the motivic cohomology groups (Proposition 2.4).

A. Beilinson ([2]) and S. Lichtenbaum ([6]) proposed the following conjecture which generalizes Bloch-Kato conjecture in weight $n$.

Conjecture 5.6 The morphism

$$
\mathbf{Z} / l(n) \rightarrow B / l(n)
$$

is a quasi-isomorphism of complexes of sheaves in Zariski topology on $\mathrm{Sm} / \mathrm{k}$. In particular for any scheme of finite type over $k$ there are canonical isomorphisms:

$$
H^{i}(X, \mathbf{Z} / l(n)) \cong H^{i}(X, B / l(n))
$$

We have the following analogs of Lemmas 4.3, 4.4.

Lemma 5.7 Let $k$ be a which admits resolution of singularities field such that Beilinson-Lichtenbaum conjecture holds over $k$ in weight $n$. Then it holds over $k$ in all weights less than $n$.

Proof: The proof is exactly the same as for Lemma 4.3 with the only difference being that we use localization theorem for motivic cohomology instead of the localization theorem for Milnor's K-theory which requires the resolution of singularities assumption.

Lemma 5.8 Let $k$ be a field and $k^{\prime}=k(\xi)$ where $\xi$ is a primitive $l$-th root of unit. Then Beilinson-Lichtenbaum conjecture holds in weight $n$ over $k$ if and only if it holds in weight $n$ over $k^{\prime}$.

Proof: Same as for Lemma 4.4.

At that moment Beilinson-Lichtenbaum conjecture is verified in the following cases: 
1. It is true for $n=0,1$ by Proposition 2.2 and theorem Hilbert 90 .

2. The homomorphisms

$$
H^{i}(X, \mathbf{Z} / l(n)) \rightarrow H^{i}(X, B / l(n))
$$

are isomorphisms for any $n \geq \operatorname{dim}(X)$ if $X$ is a scheme of finite type over an algebraically closed field which admits resolution of singularities (see $[12])$.

We are ready now to state our main theorem.

Theorem 5.9 Let $k$ be a field which admits resolution of singularities and suppose that Bloch-Kato conjecture holds over $k$ in weight $n$. Then BeilinsonLichtenbaum conjecture holds over $k$ in weight $n$. In particular in this case we have;

$$
H^{i}(X, \mathbf{Z} / l(n))=0
$$

for $i<0$ and all schemes $X$ of finite type over $k$.

The following proposition shows that to prove Beilinson-Lichtenbaum conjecture it is sufficient to consider motivic cohomology of fields.

Proposition 5.10 Suppose that for any field $F$ of finite type over $k$ the homomorphisms

$$
H^{i}(\operatorname{Spec}(F), \mathbf{Z} / l(n)) \rightarrow H_{e t}^{i}\left(F, \mu_{l}^{\otimes n}\right)
$$

are isomorphisms. Then the morphism

$$
\mathbf{Z} / l(n) \rightarrow B / l(n)
$$

is a quasi-isomorphism in Zariski topology.

Proof: Since both $\mathbf{Z} / l(n)$ and $B / l(n)$ are complexes of sheaves with transfers with homotopy invariant cohomology it follows from the fact that a morphism of such sheaves is an isomorphism if and only if it gives isomorphisms on all fields of finite type over $k$ (see [15, Prop. 4.20]).

In fact there is an even "simplier" criterion for Beilinson-Lichtenbaum conjecture. Namely one has. 
Proposition 5.11 Suppose that for all $i<n$ and all fields $F$ of finite type over $k$ the homomorphisms

$$
H^{i}(\operatorname{Spec}(F), \mathbf{Z} / l(n)) \rightarrow H_{e t}^{i}\left(F, \mu_{l}^{\otimes n}\right)
$$

are injective and the homomorphisms

$$
H^{n}(\operatorname{Spec}(K), \mathbf{Z} / l(n)) \rightarrow H_{e t}^{n}\left(K, \mu_{l}^{\otimes n}\right)
$$

are isomorphisms. Then Beilinson-Lichtenbaum conjecture holds over $k$ in weight $n$.

Proof: Since Beilinson-Lichtenbaum conjecture holds for weight zero we may assume by induction that under the assumption of the proposition it holds for all weights less than $n$.

In view of Proposition 5.11 it is sufficient to show that for any field $F$ of finite type over $k$ and any $i<n$ the homomorphism

$$
H^{i}(\operatorname{Spec}(F), \mathbf{Z} / l(n)) \rightarrow H_{e t}^{i}\left(F, \mu_{l}^{\otimes n}\right)
$$

is surjective. By Lemma 5.8 we may assume that $k$ contains a primitive m-th root of unit $\xi$.

By Proposition 2.2 we have

$$
H^{0}(k, \mathbf{Z} / l(1))=H^{0}(k, B / l(1))=\mu_{l} .
$$

Multiplication with $\xi$ gives us morphisms in the derived category of sheaves with transfers:

$$
\begin{aligned}
a_{\xi}: \mathbf{Z} / l(n-1) & \rightarrow \mathbf{Z} / l(n) \\
b_{\xi}: B / l(n-1) & \rightarrow B / l(n)
\end{aligned}
$$

such that the diagram

$$
\begin{array}{ccc}
\mathbf{Z} / l(n-1) & \rightarrow & \mathbf{Z} / l(n) \\
\downarrow & & \downarrow \\
B / l(n-1) & \rightarrow & B / l(n)
\end{array}
$$

commutes. It remains to notice that for any field $F$ over $k$ and any $i<n$ the homomorphisms

$$
H^{i}(F, B / l(n-1))=H_{e t}^{i}\left(F, \mu_{l}^{\otimes(n-1)}\right) \stackrel{b_{\xi}}{\rightarrow} H_{e t}^{i}\left(F, \mu_{l}^{\otimes n}\right)=H^{i}(F, B / l(n))
$$

are isomorphisms. 


\section{Main theorem.}

In this section we prove Theorem 5.9.

Denote by $\partial \Delta^{j}$ the closed subscheme in $\mathbf{A}^{j+1}$ given by the equations

$$
\begin{aligned}
& \sum_{i=0}^{j} x_{i}=1 \\
& \prod_{i=0}^{j} x_{i}=0 .
\end{aligned}
$$

The scheme $\partial \Delta^{j}$ has a closed covering by $j+1$ closed subschemes isomorphic to $\mathbf{A}^{j-1}$. We denote by $p_{0}, \ldots, p_{j}$ the "vertices" of $\partial \Delta^{j}$, i.e. $p_{i}$ is the point given by the equations $x_{k}=0$ for $k \neq i$.

Lemma 6.1 For all $j \geq 1$ there are canonical isomorphisms:

$$
\begin{aligned}
& H^{i}\left(\partial \Delta^{j}, \mathbf{Z} / l(n)\right)=H^{i}(k, \mathbf{Z} / l(n)) \oplus H^{i-j+1}(k, \mathbf{Z} / l(n)) \\
& H^{i}\left(\partial \Delta^{j}, B / l(n)\right)=H^{i}(k, B / l(n)) \oplus H^{i-j+1}(k, B / l(n))
\end{aligned}
$$

compatible with homomorphisms

$$
H^{*}(-, \mathbf{Z} / l(n)) \rightarrow H^{*}(-, B / l(n)) .
$$

Proof: We will only consider the first isomorphism. The proof of the second one is exactly the same. The direct summand $H^{i}(k, \mathbf{Z} / l(n))$ is the image of the homomorphism induced by the structural projection

$$
\partial \Delta^{j} \rightarrow \operatorname{Spec}(k)
$$

which splits by the vertex $p_{0}$.

We proceed now by induction by $j$. For $j=1$ the scheme $\partial \delta^{j}$ is the disjoint union of vertices $p_{0}$ and $p_{1}$ which proves the lemma in this case.

Consider the closed covering of $\partial \Delta^{j}$ by the subschemes $V_{j}, W_{j}$ given by the equations

$$
x_{j}=0
$$

and

$$
\prod_{k=0}^{j-1} x_{k}=0
$$


respectively. The scheme $V_{j} \cap W_{j}$ is isomorphic to $\partial \Delta^{j-1}$. Thus by Corollary 2.8 and the inductive assumption we have a long exact sequence of the form

$$
\begin{gathered}
\ldots \rightarrow H^{i-1}\left(W_{j}, \mathbf{Z} / l(n)\right) \oplus H^{i-1}\left(V_{j}, \mathbf{Z} / l(n)\right) \rightarrow H^{i}(k, \mathbf{Z} / l(n)) \oplus H^{i-j+1}(k, \mathbf{Z} / l(n)) \rightarrow \\
\rightarrow H^{i}\left(\partial \Delta^{j}, \mathbf{Z} / l(n)\right) \rightarrow H^{i}\left(W_{j}, \mathbf{Z} / l(n)\right) \oplus H^{i}\left(V_{j}, \mathbf{Z} / l(n)\right) \rightarrow \ldots
\end{gathered}
$$

It remains to observe that both $V_{j}$ and $W_{j}$ are contractible and since motivic cohomology are homotopy invariant we have:

$$
\begin{aligned}
& H^{i}\left(W_{j}, \mathbf{Z} / l(n)\right)=H^{i}(k, \mathbf{Z} / l(n)) \\
& H^{i}\left(W_{j}, \mathbf{Z} / l(n)\right)=H^{i}(k, \mathbf{Z} / l(n))
\end{aligned}
$$

We will say that an element $\alpha$ in $H^{i}\left(\partial \Delta^{j}, \mathbf{Z} / l\right)$ is a reduced element if it belongs to the subgroup $H^{i-j+1}(k, \mathbf{Z} / l)$ under the decomposition of Lemma 6.1. Clearly this condition holds if and only if the restriction of $\alpha$ to a vertex of $\partial \Delta^{j}$ is zero.

Denote by $\psi_{n}$ the obvious canonical element in $H^{n}\left(\left(\mathbf{A}^{1}-\{0\}\right)^{n}, \mathbf{Z} / l(n)\right)$.

Lemma 6.2 Let $\alpha$ be a reduced element in $H^{n}\left(\partial \Delta^{j}, \mathbf{Z} / l(n)\right)$. Then there exists a finite morphism $Z \rightarrow \partial \Delta^{j}$, a relative cycle $\mathcal{Z}$ in $c\left(Z / \partial \Delta^{j}, 0, \mathbf{Z} / l\right)$ and a morphism $f: Z \rightarrow\left(\mathbf{A}^{1}-\{0\}\right)^{n}$ such that

$$
\alpha=\left(\mathcal{Z}, f^{*}\left(\psi_{n}\right)\right)
$$

where $(-,-)$ is the pairing introduced in Proposition 2.9.

Proof: By Lemma 6.1 the element $\alpha$ corresponds to an element $\alpha^{\prime} \in H^{n-j+1}(k, \mathbf{Z} / l(n))$. By definition motivic cohomology of a field $k$ are homology groups of a direct summand of the complex $\underline{C}_{*}\left(L\left((\mathbf{A}-\{0\})^{n}\right) \otimes\right.$ $\mathbf{Z} / l$ ). In particular $\alpha^{\prime}$ can be represented by an element in

$$
\underline{C}_{j-1}\left(L\left((\mathbf{A}-\{0\})^{n}\right) \otimes \mathbf{Z} / l\right)=L\left((\mathbf{A}-\{0\})^{n}\right)\left(\Delta^{j-1}\right) \otimes \mathbf{Z} / l
$$

whose restriction to all faces of $\Delta^{j-1}$ is zero. Again by definition such an element is a cycle $\mathcal{Z}$ (with $\mathbf{Z} / l$-coefficients) in $\Delta^{j-1} \times(\mathbf{A}-\{0\})^{n}$ which is a formal linear combination of closed subsets which are finite and surjective over $\Delta^{j-1}$. Let $Z$ be the union of this closed subsets considered as a reduced 
scheme finite over $\Delta^{j-1}$ and $f: Z \rightarrow(\mathbf{A}-\{0\})^{n}$ be the obvious morphism. We may consider $\Delta^{j-1}$ as one of the faces of $\partial \Delta^{j}$. It is easy to see that the condition on $\mathcal{Z}$ saying that its restriction to all faces of $\Delta^{j-1}$ is zero is equivalent to the fact that considered as a cycle on $Z$ it belongs to $c\left(Z / \partial \Delta^{j}, 0, \mathbf{Z} / l\right)$. It follows now from the construction of transfers in motivic cohomology that we have

$$
\alpha=\left(\mathcal{Z}, f^{*}\left(\psi_{n}\right)\right)
$$

which proves the lemma.

Lemma 6.3 Let $\alpha$ be a reduced element of $H^{n}\left(\partial \Delta^{j}, \mathbf{Z} / l(n)\right)$. Then there exists an open subset $U$ of $\partial \Delta^{j} \times \mathbf{A}^{1}$ which contains all the points of the form $p_{i} \times\{0\}, p_{i} \times\{1\}$ and an element $\beta \in H^{n}(U, \mathbf{Z} / l(n))$ such that its restriction to $\partial \Delta^{j} \times\{0\} \cap U$ equals $\alpha_{\mid U}$ and its restriction to $\partial \Delta^{j} \times\{1\} \cap U$ equals zero.

Proof: By Lemma 6.2 there is a finite surjective morphism $Z \rightarrow \partial \Delta^{j}$, a relative cycle $\mathcal{Z}$ in $c\left(Z / \partial \Delta^{j}, 0, \mathbf{Z} / l\right)$ and a morphism $f: Z \rightarrow\left(\mathbf{A}^{1}-\{0\}\right)^{n}$ such that $\alpha=\left(\mathcal{Z}, f^{*}\left(\psi_{n}\right)\right)$. Consider the finite morphism $q: Z \times \mathbf{A}^{1} \rightarrow \partial \Delta^{j} \times \mathbf{A}^{1}$ and let $\mathcal{Y}$ be the relative cycle $\operatorname{cycl}(p r)(\mathcal{Z})$ on $Z \times \mathbf{A}^{1}$ over $\partial \Delta^{j} \times \mathbf{A}^{1}$ where

$$
p r: \partial \Delta^{j} \times \mathbf{A}^{1} \rightarrow \partial \Delta^{j}
$$

is the projection.

Consider the morphism $Z \times\{0\} \amalg Z \times\{1\} \rightarrow\left(\mathbf{A}^{1}-\{0\}\right)^{n}$ which equals $f$ on the first component and equals $(1, \ldots, 1)$ on the second. Since the morphism $q$ is finite there is an open neighborhood $U$ of points $p_{i} \times\{0\}$, $p_{i} \times\{1\}$ on $\partial \Delta^{j} \times \mathbf{A}^{1}$ and a morphism $f^{\prime}: q^{-1}(U) \rightarrow\left(\mathbf{A}^{1}-\{0\}\right)^{n}$ such that its restriction to $q^{-1}(U) \cap(Z \times\{0\} \amalg Z \times\{1\})$ equals $f$. We set:

$$
\beta=\left(\mathcal{Y}_{\mid U},\left(f^{\prime}\right)^{*}\left(\psi_{n}\right)\right) \text {. }
$$

The properties of $\beta$ required by the conditions of our lemma follow immediately from Proposition 2.9 .

Let $k$ be a field which admits resolution of singularities and $X$ be a scheme of finite type over $k$. We define strictly dense open subschemes in $X$ by the following rule: 
1. An open subscheme in a smooth scheme is strictly dense if and only if it is dense.

2. An open subscheme $U$ in a scheme of finite type $X$ is strictly dense if there exists a resolution of singularities $p: \tilde{X} \rightarrow X$ such that $p^{-1}(U)$ is dense in $\tilde{X}$ and $p^{-1}(U) \cap p^{-1}\left(X_{\text {sing }}\right)$ is strictly dense in $p^{-1}\left(X_{\text {sing }}\right)$ where $X_{\text {sing }}$ is the closed subset of singular points of $X$.

As an example consider the case of a scheme $X$ which has a closed covering $X=\cup X_{i}$ such that $X_{i}$ and all the intersections $X_{i_{1}} \cap \ldots \cap X_{i_{k}}$ are smooth. Then an open subscheme $U$ in $X$ is strictly dense if and only if for any $i_{1}, \ldots, i_{k}$ the intersection $X_{i_{1}} \cap \ldots \cap X_{i_{k}} \cap U$ is dense in $X_{i_{1}} \cap \ldots \cap X_{i_{k}}$.

Lemma 6.4 Let $k$ be a field which admits resolution of singularities and such that Beilinson-Lichtenbaum conjecture holds over $k$ in weights less than $n$. Let further $X$ be a scheme of finite type over $k, U$ be a strictly dense open subscheme in $X$ and $Z=X-U$. Then the canonical homomorphisms of cohomology with supports

$$
H_{Z}^{i}(X, \mathbf{Z} / l(n)) \rightarrow \mathbf{H}_{Z}^{i}(X, B / l(n))
$$

are isomorphisms.

Proof: It is easy to see from the definition of a strictly dense open subscheme and the blow-up exact sequences (Propositions 2.7,5.2) for motivic cohomology and cohomology with coefficients in $B / l(n)$ respectively that it is sufficient to prove our lemma for a smooth connected scheme $X$.

In this case it follows easily by induction on $\operatorname{dim}(X-U)$ from Propositions $2.4,5.5$.

We will use the following important fact.

Theorem 6.5 Let $k$ be a field, $S$ be the semi-local scheme of a finite set of points on a scheme of finite type over $k$ and $\alpha \in H_{\text {et }}^{i}\left(S, \mu_{l}^{\otimes n}\right)$ be an etale cohomology class. Then there exists a smooth scheme $X$ over $k$, a morphism $f: S \rightarrow X$ and an etale cohomology class $\beta \in H_{e t}^{i}\left(X, \mu_{l}^{\otimes n}\right)$ such that

$$
\alpha=f^{*}(\beta)
$$


Corollary 6.6 Let $k$ be a field such that Bloch-Kato conjecture holds over $k$ in weight $n$. Let $S$ be the semi-local scheme of a finite set of points on a scheme of finite type over $k$. Then the canonical homomorphism

$$
H^{n}(S, \mathbf{Z} / l(n)) \rightarrow H^{n}(S, B / l(n))
$$

is surjective.

Proof: The right hand side group is isomorphic to $H_{e t}^{n}\left(S, \mu_{l}^{\otimes n}\right)$ by Proposition 5.3. Thus by Theorem 6.5 we may assume $S$ to be a smooth semi-local scheme. Consider the canonical morphism of complexes

$$
\mathbf{Z} / l(n) \rightarrow B / l(n) .
$$

Under our assumptions the morphism of cohomology sheaves

$$
\underline{H}^{n}(\mathbf{Z} / l(n)) \rightarrow \underline{H}^{n}(B / l(n))
$$

is surjective. The kernel of this homomorphism is a homotopy invariant presheave with transfers and therefore positive cohomology groups of $S$ with coefficients in it are zero. Since $\underline{H}^{i}$ on both sides is zero for $i>n$ it proves the proposition.

Proof of Theorem 5.9: In view of Proposition 5.11 it is sufficient to show that for any field $F$ of finite type over $k$ and any $i \leq n$ the homomorphism

$$
H^{i}(\operatorname{Spec}(F), \mathbf{Z} / l(n)) \rightarrow H^{i}(\operatorname{Spec}(F), B / l(n))
$$

is injective. By induction and Lemma 4.3 we may assume that BeilinsonLichtenbaum conjecture holds over $k$ for weights less than $n$.

By Lemma 6.1 it is sufficient to prove that the canonical homomorphisms

$$
\phi_{j}: H^{n}\left(\partial \Delta_{F}^{j}, \mathbf{Z} / l(n)\right) \rightarrow \mathbf{H}^{n}\left(\partial \Delta_{F}^{j}, B / l(n)\right)
$$

are monomorphisms for all $j$.

Let $S^{1}$ be the scheme obtained from $\mathbf{A}^{1}$ by gluing together point $\{0\}$ and $\{1\}$. Denote the distinguished point on $S^{1}$ by $x$. One can easily see using Propositions 2.7, 5.2 that there are monomorphisms of the form

$$
H^{n}\left(\partial \Delta_{F}^{j}, \mathbf{Z} / l(n)\right) \rightarrow H^{n+1}\left(\partial \Delta_{F}^{j} \times S^{1}, \mathbf{Z} / l(n)\right)
$$




$$
H^{n}\left(\partial \Delta_{F}^{j}, B / l(n)\right) \rightarrow H^{n+1}\left(\partial \Delta_{F}^{j} \times S^{1}, B / l(n)\right) .
$$

Lemma 6.3 implies that for any reduced element $\alpha$ in $H^{n}\left(\partial \Delta_{F}^{j}, \mathbf{Z} / l(n)\right)$ there is an open neighborhood $U$ of the points $p_{0} \times\{x\}, \ldots, p_{j} \times\{x\}$ in $\partial \Delta_{F}^{j} \times S^{1}$ such that the image of $\alpha$ in $H^{n}(U, \mathbf{Z} / l(n))$ is zero.

Denote by $S$ the semi-local scheme of the set $\left\{p_{0} \times\{x\}, \ldots, p_{j} \times\{x\}\right\}$. For any complex of sheaves $K$ denote further by $H_{\mathcal{Z}}^{*}\left(\partial \Delta_{F}^{j} \times S^{1}, K\right)$ the direct limit of hypercohomology with supports

$$
H_{\mathcal{Z}}^{*}\left(\partial \Delta_{F}^{j} \times S^{1}, K\right)=\lim _{Z} H_{Z}^{*}\left(\partial \Delta_{F}^{j}, K\right)
$$

where $Z$ runs through the set of closed subschemes in $\partial \Delta_{F}^{j} \times S^{1}$ such that

$$
Z \cap\left\{p_{0} \times\{x\}, \ldots, p_{j} \times\{x\}\right\}=\emptyset .
$$

Note that the open subschemes of the form $X_{Z}$ for such closed subsets $Z$ are exactly the strictly dense open subschemes in $\partial \Delta_{F}^{j} \times S^{1}$.

We have a morphism of long exact sequences of the form:

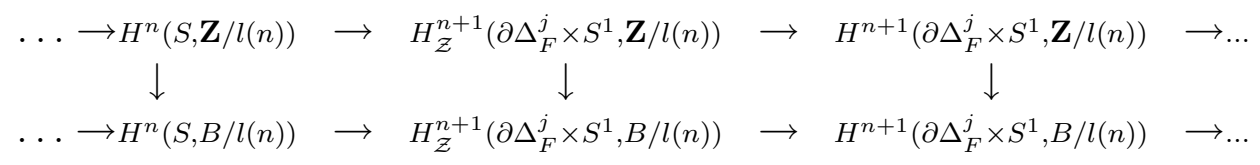

By Lemma 6.4 and the inductive assumption the middle vertical arrow is an isomorphism. By Corollary 6.6 the left hand side arrow is an epimorphism and our theorem follows now by the standard diagram search.

\section{$7 \quad$ Some applications.}

In this section we give some applications of Theorem 5.9. We start with the following proposition which shows that Bloch-Kato conjecture is closely related to vanishing of Bokstein homomorphisms in etale cohomology of fields.

Proposition 7.1 Bloch-Kato conjecture holds over $k$ in weight $n$ if and only if for any field $F$ of finite type over $k$ the Bokstein homomorphisms

$$
\beta_{n, m}: H_{e t}^{n}\left(F, \mu_{l^{m}}^{\otimes n}\right) \rightarrow H_{e t}^{n+1}\left(F, \mu_{l}^{\otimes n}\right)
$$

are zero for all $m>0$. 
Proof: Let us show first that if Bloch-Kato conjecture holds over $k$ in weight $n$ then the Bokstein homomorphisms $\beta_{n, m}$ are zero. In fact Bloch-Kato conjecture implies that any element in $H_{e t}^{n}\left(F, \mu_{l m}^{\otimes n}\right)$ is a sum of products of elements from $H_{e t}^{1}\left(F, \mu_{l^{m}}\right)$. Since Bokstein homomorphisms behave well with respect to the multiplicative structure on cohomology it remains to show that

$$
\beta_{1}: H_{e t}^{1}\left(F, \mu_{l^{m}}\right) \rightarrow H_{e t}^{2}\left(F, \mu_{l}\right)
$$

is zero. It is a well known fact which follows trivially from Theorem Hilbert 90.

Assume now that $\beta_{n, m}=0$ for all fields $F$ of finite type over $k$. By the standard argument it implies that the same holds for all $\beta_{n^{\prime}, m}$ with $n^{\prime}<n$ and therefore we may assume by induction and Theorem 5.9 that Bloch-Kato conjecture holds over $k$ in weights less than $n$. It remains to show that under these assumptions the homomorphisms

$$
K_{n}^{M}(F) \rightarrow H_{e t}^{n}\left(F, \mu_{l}^{\otimes n}\right)
$$

are surjective. Note first that under our assumption $H_{e t}^{n}\left(F, \mu_{l}^{\otimes n}\right)$ is the ltorsion subgroup in $H_{e t}^{n}\left(F, \mathbf{Q}_{l} / \mathbf{Z}_{l}(n)\right)$ (where $\mathbf{Q}_{l} / \mathbf{Z}_{l}(n)=\lim \mu_{l^{m}}^{\otimes n}$ ) and that the later group is infinitely divisible.

Consider the homomorphism

$$
K_{n}^{M}(F) \otimes \mathbf{Q}_{l} / \mathbf{Z}_{l} \rightarrow H_{e t}^{n}\left(F, \mathbf{Q}_{l} / \mathbf{Z}_{l}(n)\right) .
$$

Let us show that it is a surjection. We need the following trivial lemma.

Lemma 7.2 Let $F$ be a field and $\alpha$ be an element in $H_{e t}^{n}\left(F, \mathbf{Q}_{l} / \mathbf{Z}_{l}(n)\right)$. Then there exists a regular connected variety $U$ over $F$, a pair of $F$-points $x_{0}, x_{1}$ : $\operatorname{Spec}(F) \rightarrow U$ and a cohomology class $\alpha^{\prime} \in H_{e t}^{n}\left(U, \mathbf{Q}_{l} / \mathbf{Z}_{l}(n)\right)$ such that

$$
\begin{gathered}
x_{0}^{*}\left(\alpha^{\prime}\right)=0 \\
x_{1}^{*}\left(\alpha^{\prime}\right)=\alpha .
\end{gathered}
$$

Moreover the variety $U$ can be chosen in such a way that it has an etale Galois covering $p: \tilde{U} \rightarrow U$ by a rational variety over $F$ and $x_{0}$ lifts to an F-point $\tilde{x}_{0}$ of $\tilde{U}$. 
Proof: Let $\operatorname{Spec}\left(F^{\prime}\right) \rightarrow \operatorname{Spec}(F)$ be a finite Galois extension which realizes $\alpha$. Let further $U_{G}$ be the open subscheme of regular points in $\mathbf{A}^{\# G} / G$ where $G$ acts on $\mathbf{A}^{\# G}$ by obvious permutations of coordinates. One can easily see that there is a point $x_{1}$ on $U$ and a class $\alpha^{\prime} \in H_{e t}^{n}\left(U, \mathbf{Q}_{l} / \mathbf{Z}_{l}(n)\right)$ which can be realized by the canonical Galois covering of $U$ with the Galois group $G$ such that $x_{1}^{*}\left(\alpha^{\prime}\right)=\alpha$. Moreover since the preimage of $U$ in $\mathbf{A}^{\# G}$ has $F$-rational points there is another point $x_{0}$ on $U$ such that $x_{0}^{*}\left(\alpha^{\prime}\right)=0$.

Let $\alpha$ be an element of $H_{e t}^{n}\left(F, \mathbf{Q}_{l} / \mathbf{Z}_{l}(n)\right)$ and $p: \tilde{U} \rightarrow U, x_{0}, x_{1}, \alpha^{\prime}$ be as in Lemma 7.2. Denote by $d$ the degree of $p$. Since the group $H_{e t}^{n}\left(F, \mathbf{Q}_{l} / \mathbf{Z}_{l}(n)\right)$ is infinitely divisible we can find an element $\gamma \in H_{e t}^{n}\left(U, \mathbf{Q}_{l} / \mathbf{Z}_{l}(n)\right)$ such that

$$
\begin{gathered}
x_{0}^{*}(\gamma)=0 \\
d \gamma=\alpha^{\prime} .
\end{gathered}
$$

Then we have $\alpha^{\prime}=p_{*}\left(p^{*}(\gamma)\right)$ and it remains to show that $p^{*}(\gamma)$ belongs to the image of the homomorphism

$$
H_{\mathcal{M}}^{n}\left(\tilde{U}, \mathbf{Q}_{l} / \mathbf{Z}_{l}(n)\right) \rightarrow H_{e t}^{n}\left(\tilde{U}, \mathbf{Q}_{l} / \mathbf{Z}_{l}(n)\right) .
$$

It follows from the lemma below and the inductive assumption:

Lemma 7.3 Let $U$ be a smooth connected rational variety over $F$ and $x$ be an F-point of $U$. Assume that Bloch-Kato conjecture holds over $k$ in weights less that $n$. Then the homomorphism

$$
\tilde{H}_{\mathcal{M}}^{n}\left(U, \mathbf{Q}_{l} / \mathbf{Z}_{l}(n)\right) \rightarrow \tilde{H}_{e t}^{n}\left(U, \mathbf{Q}_{l} / \mathbf{Z}_{l}(n)\right),
$$

where $\tilde{H}$ denotes the group of classes which vanish on $x$, is an isomorphism.

Proof: It is sufficient to consider the case of $\mathbf{Z} /$ l-coefficients. Due to localization theorems 2.4, 5.5 and our assumption that the Bloch-Kato conjecture holds in weights less than $n$ we may replace $U$ by any variety which is birationally equivalent to it. Thus we may assume that $U=\mathbf{A}^{N}$. In this case the lemma holds since both left and right hand side groups are zero by the homotopy invariance property of the corresponding theories.

To finish the proof of our proposition it remains to note that the technique we used to prove Theorem 5.9 works for $\mathbf{Q}_{l} / \mathbf{Z}_{l}$-coefficients as well as for $\mathbf{Z} / l$ coefficients which implies that the homomorphisms

$$
K_{n}^{M}(F) \otimes \mathbf{Q}_{l} / \mathbf{Z}_{l} \rightarrow H_{e t}^{n}\left(F, \mathbf{Q}_{l} / \mathbf{Z}_{l}(n)\right)
$$


are in fact isomorphisms. Therefore the same holds for the subgroups of l-torsion elements in them.

The following proposition explicitly describes all motivic cohomology of smooth schemes of weight 2 with finite coefficients and in weight 3 with $\mathbf{Z} / 2$-coefficients.

Note that due to the result of [12] we have

$$
H^{i}(X, \mathbf{Z} / l(n))=C H^{n}(X, 2 n-i, \mathbf{Z} / l)
$$

where the groups on the right hand side are the higher Chow groups defined by S. Bloch ([3]). Thus in particular we get a description of all higher Chow groups of smooth varieties in codimension 2 (all finite coefficients) and in codimension 3 ( $\mathbf{Z} / 2$-coefficients).

Proposition 7.4 Let $X$ be a smooth variety over a field $k$ which admits resolution of singularities and $m$ be an integer prime to char $(k)$. Then one has:

$$
H^{i}(X, \mathbf{Z} / m(2))= \begin{cases}0 & \text { for } i<0 \\ H_{e t}^{i}\left(X, \mu_{m}^{\otimes 2}\right) & \text { for } i=0,1,2 \\ k e r\left(H_{e t}^{3}\left(X, \mu_{m}^{\otimes 2}\right) \rightarrow H_{e t}^{3}\left(k(X), \mu_{m}^{\otimes 2}\right)\right) & \text { for } i=3 \\ A^{2}(X) / m & \text { for } i=4 \\ 0 & \text { for } i>4\end{cases}
$$

Similarly if char $(k) \neq 2$ and $m=2^{n}$ one has:

$H^{i}(X, \mathbf{Z} / m(3))= \begin{cases}0 & \text { for } i<0 \\ H_{e t}^{6-r}\left(X, \mu_{m}^{\otimes 3}\right) & \text { for } r=0,1,2,3 \\ k e r\left(H_{e t}^{4}\left(X, \mu_{m}^{\otimes 3}\right) \rightarrow H_{e t}^{4}\left(k(X), \mu_{m}^{\otimes 3}\right)\right) & \text { for } i=4 \\ H^{5}(X, B / m(3)) & \text { for } r=5 \\ A^{3}(X) / m & \text { for } i=6 \\ 0 & \text { for } i>6\end{cases}$

where $A^{i}(X)$ is the group of cycles of codimension $i$ on $X$.

Proof: It follows immediately from Theorem 5.9 and the fact that BlochKato conjecture holds in weight 2 and in weight 3 for $\mathbf{Z} / 2$-coefficients. 
Proposition 7.5 Let $k$ be an algebraically closed field which admits resolution of singularities, $l$ be an integer prime to char $(k)$ and $X$ be a scheme of finite type over $k$. Suppose that one of the following conditions holds:

1. $\operatorname{dim}(X) \leq 3$.

2. $\operatorname{dim}(X) \leq 4$ and $l=2^{n}$.

Then for all $i, n \in \mathbf{Z}$ the homomorphisms

$$
H^{i}(X, \mathbf{Z} / l(n)) \rightarrow H^{i}(X, B / l(n))
$$

are isomorphisms, i.e. Beilinson-Lichtenbaum conjecture holds for all $X$ and $l$ as above.

Proof: Since the Bloch-Kato conjecture is proven in weights $\leq 2$ and i weight 3 for $\mathbf{Z} / 2$-coefficients we have only to consider the case $n=\operatorname{dim}(X)$. Since there is a canonical homomorphism

$$
H^{i}(X, \mathbf{Z} / l(n)) \rightarrow H^{i}(X, B / l(n))
$$

and the groups on both sides have long exact sequences with respect to blowups we may assume that $X$ is smooth. Consider first the case $i \leq n$. Then the right hand side group is isomorphic to $H_{e t}^{i}\left(X, \mu_{l}^{\otimes n}\right)$ and the required isomorphism

$$
H^{i}(X, \mathbf{Z} / l(n)) \rightarrow H_{e t}^{i}\left(X, \mu_{l}^{\otimes n}\right)
$$

was established in [12]. The general result follows no from the fact that both left and right hand side groups vanish for $i>n$ for local smooth schemes.

Corollary 7.6 Let $k$ be an algebraically closed field which admits resolution of singularities, $l$ be an integer prime to char $(k)$ and $X$ be a scheme of finite type over $k$. Suppose that one of the following conditions holds:

1. $\operatorname{dim}(X) \leq 3$.

2. $\operatorname{dim}(X) \leq 4$ and $l=2^{n}$.

Then the Quillen-Lichtenbaum conjecture holds for X, i.e. the canonical homomorphisms

$$
K_{i}(X, \mathbf{Z} / l) \rightarrow K_{i, e t}(X, \mathbf{Z} / l)
$$

are isomorphisms for $i \geq \operatorname{dim}(X)-1$ 
Proof: Since both for algebraic and for the etale K-theories there are BrownGersten spectral sequences it is sufficient to consider the case when $X$ is the spectrum of a field of transcendental degree $\leq 3$ ( $\leq 4$ for $\mathbf{Z} / 2$-coefficients) over $k$. In this case we have a motivic spectral sequence constructed in [4] which converges from higher Chow groups to the algebraic K-theory and the standard spectral sequence which converges from etale cohomology to the etale K-theory. Therefore, the required result follows from Proposition 7.5.

Remark: The same kind of argument together with Theorem 5.9 shows that the Bloch-Kato conjecture in weight $n$ implies Quillen-Lichtenbaum conjecture for varieties of dimension $n+1$ over algebraically closed fields with resolution of singularities.

\section{References}

[1] H. Bass and J. Tate. The Milnor ring of a global field. In K-theory II, volume 342 of Lecture Notes in Math., pages 349-446. Springer-Verlag, 1973.

[2] A. Beilinson. Height pairing between algebraic cycles. In K-theory, Arithmetic and Geometry., volume 1289 of Lecture Notes in Math., pages 1-26. Springer-Verlag, 1987.

[3] S. Bloch. Algebraic cycles and higher K-theory. Adv. in Math., 61:267304, 1986.

[4] S. Bloch and S. Lichtenbaum. A spectral sequence for motivic cohomology. Preprint, 1994.

[5] E. Friedlander and V. Voevodsky. Bivariant cycle cohomology. Cycles, transfers and motivic homology theories, page ..., 1995.

[6] S. Lichtenbaum. Values of zeta-functions at non-negative integers. In Number theory, volume 1068 of Lecture Notes in Math., pages 127-138. Springer-Verlag, 1983.

[7] A. Merkurjev and A. Suslin. K-cohomology of Severy-Brauer varieties and the norm residue homomorphism. Math. USSR Izvestiya, 21:307340, 1983. 
[8] A. Merkurjev and A. Suslin. The norm residue homomorphism of degree three. Math. USSR Izvestiya, 36(2):349-367, 1991.

[9] Yu. P. Nesterenko and A.Suslin. Homology of the general linear group over a local ring and Milnor K-theory. Izv. AN SSSR.

[10] M. Rost. Hilbert 90 for $K_{3}$ for degree-two extensions. Preprint, 1986.

[11] A. Suslin. Algebraic K-theory and the norm residue homomorphism. $J$. Soviet Math., 30:2556-2611, 1985.

[12] A. Suslin. Higher Chow groups and etale cohomology. Cycles, transfers and motivic homology theories, page ..., 1995.

[13] A. Suslin and V. Voevodsky. Singular homology of abstract algebraic varieties. Preprint., 1992.

[14] A. Suslin and V. Voevodsky. Relative cycles and Chow sheaves. Cycles, transfers and motivic homology theories, page ..., 1995.

[15] V. Voevodsky. Cohomological theory of presheaves with transfers. $C y$ cles, transfers and motivic homology theories, page ..., 1995.

[16] V. Voevodsky. Triangulated categories of motives over a field. Cycles, transfers and motivic homology theories, page ..., 1995. 